\title{
EPITAFIOS INFANTILES: UNA TRADICIÓN LITERARIA GRECOLATINA EN LA LITERATURA INGLESA $^{1}$
}

M' Pilar Hualde Pascual

Universidad Autónoma de Madrid

O. INTRODUCCIÓN: EL EPIGRAMA FUNERARIO, DE LOS ORIGGENES A LA LITERATURA EUROPEA

Entre las más tempranas manifestaciones literarias en Grecia aparece el epigrama, pequeña composición en verso, grabada inicialmente sobre algún objeto, bien con fines votivos, bien como fórmulas de maldición, o simplemente como marca de propiedad. Dentro de los epigramas destacan los epigramas funerarios o epitafios, consistentes en su origen en breves composiciones versificadas sobre las tumbas, que indican filiación, edad y alguna característica destacada del difunto. Su forma métrica más frecuente, al igual que en los epigramas votivos, suele ser el dístico elegíaco. El epigrama en general, y el epigrama funerario en particular, manifestación artística popular en su origen, alcanzará categoría de género literario y como tal triunfará,

1 Una primera versión de este trabajo con el título de $*$ La tradición literaria del epitafio infantil: Calímaco, Marcial y algunos poetas metafísicos» se leyó como Ponencia en el I Coloquio de Estudiantes de Filología Clásica (Valdepeffas) el 5 de julio de 1989. 
sobre todo a partir de la época helenística. El epigrama se introduce en la literatura latina, donde es cultivado por los Neotéricos, pero es, sobre todo, el hispano Marcial quien consagra definitivamente el epigrama en Roma. Finalmente, el epigrama de distinto signo, como composición breve en diferentes tipos de metro, aparecerá en las literaturas europeas, sobre todo a partir del Renacimiento.

Si la muerte de un ser querido ha producido a lo largo de los siglos la expresión literaria del dolor humano, cuando el difunto es un niño el hecho tiene unas connotaciones especialmente luctuosas a las que el género epigramático no es ajeno. Así, para hacer llegar al lector el sentimiento y la compasión va a servirse de un tratamiento literario particular de los elementos que, de forma más o menos constante, aparecen en las fórmulas sepulcrales, tanto griegas como latinas, y de una serie de tópicos literarios que después tendrán su pervivencia en la tradición humanística. En esta idea, vamos a revisar, dentro de la literatura griega ( $\$ 1$ ), el epitafio dedicado al niño Nicóteles, del alejandrino Calímaco, y una serie de epitafios referentes a niños, incluidos en la Antología Palatina y, entre los autores latinos ( $\$ 2)$, los epitafios infantiles del hispano Marcial. Veremos que, pese a las diferencias entre el epigrama incisivo y realista de este último y el epigrama helenístico ${ }^{2}$, ambos tipos presentan evidentes coincidencias en el tratamiento literario de un tema común, la muerte y sepultura de un niño. Así pues, uno y otro toman elementos formulares funerarios (edad, filiación, fórmula sepulcral propiamente dicha, complementos referidos a la persona del difunto) y los elaboran en sus epigramas, orientándolos hacia la compasión y piedad por la condición infantil del difunto. No obstante, cada uno se servirá de un procedimiento propio y diferente, así, mientras el epitafio de Calímaco prefiere la extrema sencillez ${ }^{3}$, Marcial opta por la implicación personal en el hecho. Situación distinta presentan los epitafios infantiles recogidos en la Antologia Palatina, en los que la afectividad dramática se buscará por medios novedosos y alambicados, pero que presentan tópicos que triunfarán en la tradición posterior.

2 La bibliografía sobre las fuentes griegas de Marcial es abundante: H. POESCHEL, Typen aus der Anthologia Palatina und den Epigrammen Martialis, Munich, 1905; E. PERTSCH, De Valerio Martiale Graecorum Poetarum imitatore, Berlín, 1911; K. PRINZ, Martial und die griechische Epigrammatik, Wien-Leipzig, 1911; J. BRECHT, Motiv und Typengeschichte des griechischen Spottepigramms, Philologus, Suppl. 22,2, Leipzig, 1930; O. AUTORE, Marziale e l'Epigramma greco, Palermo, 1937; P. LAURENS, «Martial et l'épigramme grecque du $1^{\text {er }}$ siècle ap.J.C.», REL 43, 1965, 315-341.

3 Sobre el estudio de los métodos expresivos en este y otro de los epitafios de Calímaco, cf. J. REDONDO, «Calímaco, A.P.VII 453 y 525: Un apunte sobre el epigrama sepulcral», Habis 18-19, 1987-88, pp. 87-91. 
Finalmente (§3), y trasladándonos en el tiempo a la Inglaterra isabelina, revisaremos una serie de epitafios infantiles en los que pervive la tradición grecolatina, concretamente en los llamados poetas metafísicos, como parte de la herencia que del mundo clásico han recibido: el epitafio para Salathiel Pavy, de Ben Jonson, dos epitafios dedicados a niñas de Robert Herrick y uno de Thomas Carew de la misma temática.

\section{EPITAFIOS INFANTILES EN LA LITERATURA GRIEGa: CALIMACO Y LA antologla Palatina}

Aunque la mano popular ya había creado desde antiguo interesantes epitafios inscripcionales dedicados a niños ${ }^{4}$, los primeros epitafios infantiles en la literatura griega aparecen en época helenística, dentro de la Antología Palatina y también, con un solo ejemplo claro, en Calímaco, indiscutible maestro del género epigramático ${ }^{5}$. Ciertamente, en el epitafio de Calímaco dedicado al niño Nicóteles (ep. 46), se alcanza una de las cumbres literarias del epigrama fu-

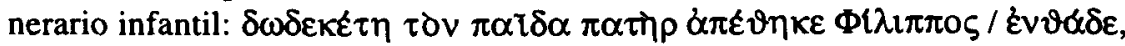

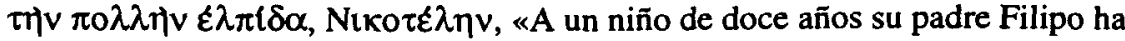
enterrado aquí. A su gran esperanza, a su Nicóteles». Se puede calificar la elaboración del poema de cuidadosamente simple, y es en su sencillez donde radica su efectividad. El epigrama consta de un sólo dístico que, en apariencia, no difiere mucho de cualquier inscripción funeraria, pero cada elemento constitutivo del epitafio ${ }^{6}$ está colocado en el lugar oportuno y produce el efecto de-

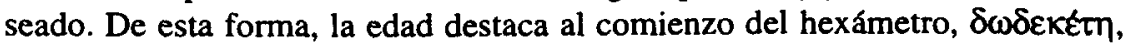
y a continuación se especifica $\tau \delta \nu \pi \alpha \tau \delta \alpha$, clave para atraer la compasión del lector, pues el enterrado sólo es un niño. La fórmula sepulcral habitual $\alpha \pi \varepsilon \vartheta \eta \kappa \varepsilon$ $\varepsilon v \vartheta ̛ \delta \delta \varepsilon$ aparece junto a la filiación, y no sólo se consigna el nombre del padre, como sería habitual en un epitafio epigráfico, sino que éste ha pasado a

4 No tratamos en este trabajo los epitafios infantiles epigráficos; nos remitimos, en cualquier caso, al excelente trabajo de M.L. DEL BARRIO como introducción de su traducción Epigramas funerarios griegos, Biblioteca Clásica Gredos, Madrid, 1992, pp.7-60, sobre todo las páginas dedicadas a la mors innarura (pp.29-32), y también al estudio de la misma autora «Función y elementos constitutivos de tos epigramas funerarios griegos», EC 95, 1989, pp. 7-20.

5 Sobre la originalidad en los epigramas de Calímaco cf. L. BRAUN, «L'arte di Calimaco negli epigrami funerari», $R E G 97,1985$, pp. 205-207.

6 Sobre los elementos constitutivos del epitafio griego cf. M." L. DEL BARRIO, art. cit., pp.7-20. 


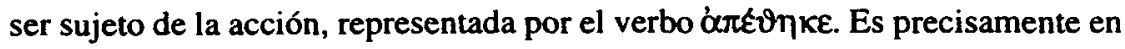
la inversión de papeles donde radica la dolorosa novedad, dado que es el padre el que tiene que sepultar al hijo y no al contrario, como sería esperable. Final-

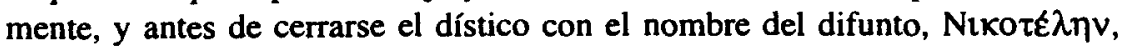

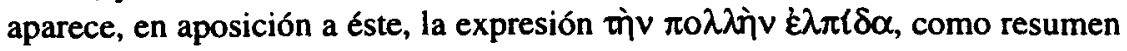
de la frustración que la pérdida de un hijo produce. Es imposible dar cuenta del dolor profundo de un padre en menor espacio y con mayor simplicidad de recursos.

Una mención aparte requieren los epigramas funerarios recogidos en la Antología Palatina, que, lejos ya de la simplicidad de los primitivos epitafios inscripcionales y de la elegante sencillez de Calímaco, se construyen con gran elaboración y artificio. La escueta fórmula sepulcral deja paso a la anécdota que, junto con la lamentación, va a ser una de las bases principales del tratamiento del tema con las que se intenta despertar la piedad del lector. En los epitafios infantiles esto se traduce, por un lado, en la descripción de muertes de niños más o menos trágicas, como la del niño Arquianacte, ahogado en un pozo ( $A P$ VII 170), la de Cleodemo, que cae por la borda de un barco ( $A P$ VII 303) o la de Coraco, que se precipita desde lo alto de una escalera (AP VII 632), hechos que constituyen la anécdota propiamente dicha. Por otra parte, producen gran efecto dramático las lamentaciones, que presentan el tema de frustración, centrado básicamente en los siguientes puntos:

(a) Referencia insistente a los vanos dolores de parto de la madre, así

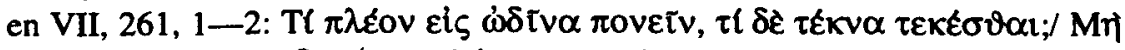

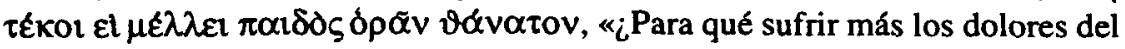
parto? ¿para que parir hijos? Que no se de a luz si ha de contemplarse la muerte de un hijo»; o a los vanos trabajos de los progenitores en general, como en

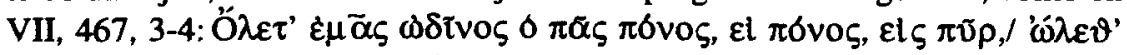

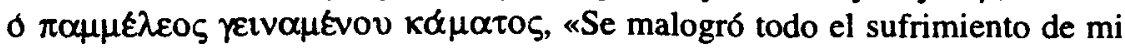
parto, si es que esto es sufrimiento, se malogró en la pira funeraria, se malogró el trabajo de tu desgraciado padre.»

(b) Lamento porque el difunto no haya podido llegar al momento del matrimonio, entendiendo por ello que no ha alcanzado la plenitud de la persona (lo que en sí constituye el tema de la mors inmatura) ${ }^{7}$, como en VII, 486,

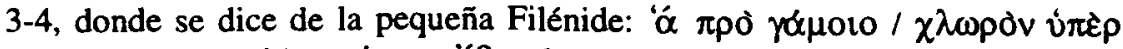

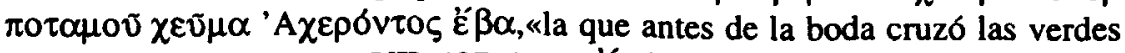

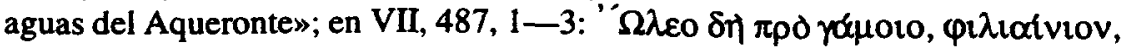

7 Frecuentísima, asimismo, en los epitafios inscripcionales (cf. M.' L. DEL BARRIO, op. cit., p. 30) e.q. W. PEEK, Griechische Versinschriften $(=G V)$ n. 683, 1450, 1680 etc. 


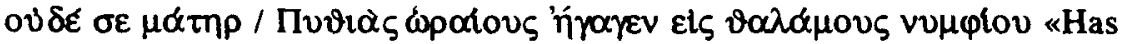
muerto, Filenion, antes de la boda, y tu madre Pitiade no ha podido conducirte en su momento al lecho de un esposo», o en VII, 488, 1-2: Aloi

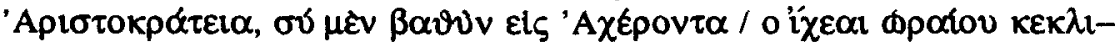

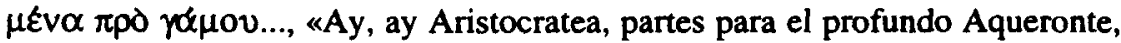
acostada antes del momento de tu boda...».

El tratamiento del tema es igual en este aspecto para jóvenes y para niños, pero la frecuente omisión de la edad hace difícil en ocasiones discernir si el difunto es un niño o un adolescente. En todo caso, cuando la edad del niño aparece explícita, forma parte también de la anécdota que procura mover a la compasión, así en $A P$ VIII, 170 y 482,VII, 643 y VII, 467. Incluso, en alguna ocasión, la determinación de la época temprana de la vida se hace mediante una perífrasis, como la referencia al niño Cleodemo VII, 303, 1: Tov $\mu i \chi \rho \delta v$

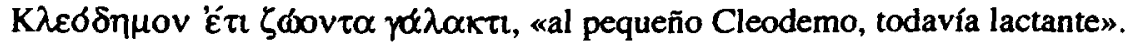

(c) Referencia a la inversion del orden natural de los hechos ${ }^{8}$, considerado explícitamente como hecho injusto en $A P$ VII, 361: Y Y $\pi$ il $\pi \alpha \tau \eta \rho \tau \delta \delta \varepsilon$

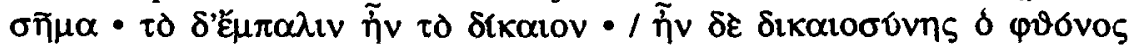

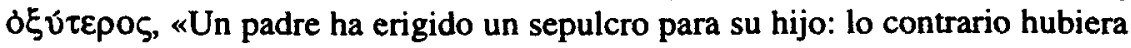
sido lo justo. Pero la muerte ha sido más rápida que la justicia».

Así, la muerte de un anciano es algo natural y obligado, pero no la de un

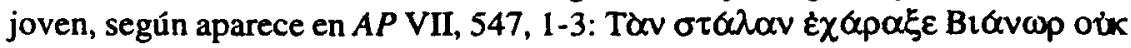

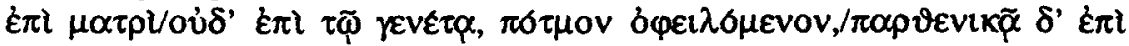
$\pi 0 r \delta l \cdot . . .$, «Bianor ha grabado esta estela, no para su madre ni su padre, cuya muerte sería natural, sino para su hija soltera».

Finalmente, es preciso señalar que ciertos tópicos funerarios, desarrollados posteriormente en la tradición del epitafio infantil, aparecen ya en la Antología Palatina:

- La súplica a la tierra para que sea leve con quien pesó poco sobre

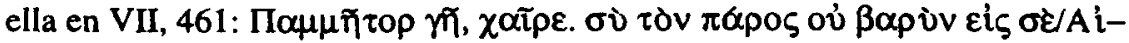

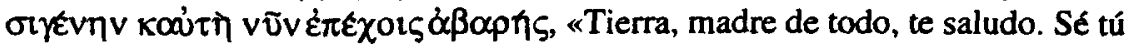
ahora leve con Esígenes, ya que él antes no fue pesado para ti». Una curiosa variante de este tema, que muestra claramente el artificio de este tipo de poemas, aparece en VII, 583, donde para un niño muerto en el parto ${ }^{9}$, ya que no la

8 También presente en epitafios inscripcionales cf. W. PEEX GV 1350, 1668 entre otros.Cf. M.' L.. DEL BARRIO, op. cit., p.31.

9 Son frecuentísimos los epitafios epigráficos de mujeres muertas en el parto, cf. M.' L. DEL BARRIO, op. cit., Pp. 30-31: el número de mujeres muertas por esta causa debía de ser muy elevado, como muestra el estudio de las tumbas en los cementerios (cf. S. B. POMEROY, Diosas, rameras, esposas y esclavas, Madrid, Akal, 1987, p. 102). 
tierra, le será ligero el propio seno materno, epitafio, como vemos, dedicado a la vez a la madre y al niño que no llegó a a nacer ${ }^{10}$ : Kov $\varphi \eta$ бol $\tau \varepsilon \lambda \varepsilon \vartheta \varepsilon \imath$

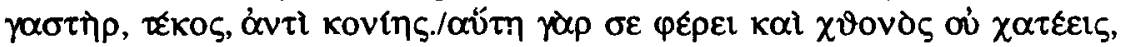
«El vientre de tu madre te es ligero, niño, en lugar del polvo, puesto que aquel te lleva y no precisas tierra».

- También será frecuente en la tradición posterior la metáfora del sueño aplicada a los niños muertos ${ }^{11}$, de lo que son un claro exponente los últimos versos de A.P. VII, 170, que presentan la sobrecogedora imagen de Arquianacte, de tres años, muerto en el regazo de su madre: $\alpha \lambda \lambda$ ' $\varepsilon \pi l$ yoúvors

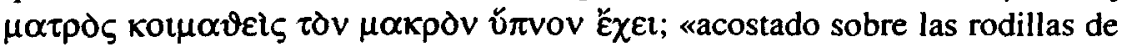
su madre duerme el sueño eterno».

2. MARCIAL Y El EPITAFIO INFANTIL EN LA LITERATURA LATINA

Los epitafios para niños compuestos por el hispano Marcial muestran una naturaleza muy distinta a los vistos en la literatura griega. Aquí el tema se desarrolla con matices personales, con la implicación del autor en el suceso, la alusión a su relación con el niño muerto y la grata evocación de las figuras infantiles (cf. Mart. Epigr. V, 34, 37; X, 61). Los elementos constantes en las fórmulas sepulcrales romanas aparecen con ciertas diferencias formales, fruto de una cuidada elaboración estilística tendente a acentuar el dramatismo de una muerte prematura:

(a) La edad en los epitafios inscripcionales romanos suele indicarse con la fórmula vixit annos (tot); a los años se añaden los meses y los días y, cuando se trata de niños, a veces hasta las horas, extremo este último no documentado en los epigramas funerarios de Marcial. Sin embargo, en estos la precisión cronológica aparece con una variación estilística, pues se indica con exactitud el tiempo que le faltó al niño para cumplir su próximo año. Así podemos verlo en el epitafio dedicado al niño Urbico, al que faltaron seis meses

10 Ya en epigramas inscripcionales se desarrolla el tema del niño muerto durante el parto o el puerperio (cf. W. PEEK GV 1024, 1606) e incluso de la mujer muerta con su hijo dentro del vientre (cf. W. PEEK $G V$ 991). También en la literatura inglesa aparece el tema del epitafio para en niño no nacido, en la obra de A. POPE (siglo XVIII), con un tratamiento totalmente distinto, pero no exento de ecos clásicos.

11 Para la asociación de la muerte con el sueño eterno en la mitología y la literatura griegas y en otras literaturas cf. M." L. DEL BARRIO, op. cit., p. 43 y n. 53, 54, 55. 
para cumplir seis años: sex mihi de prima deerant trieteride menses (Epigr. VII, 96, 3), «seis meses me faltaban para cumplir mis tres primeros años». En este caso, destaca el término trieteris en lugar de la expresión habitual annos tres. La misma idea es aplicable al conmovedor epitafio a Erotión, a quien le faltó ese mismo número de días para cumplir seis años: Impletura fuit sextae modo frigora brumae/vixisset totidem ni minus illa dies (Epigr. V, 34, 5-6), «sólo del sexto invierno iba a finar los fríos si ella hubiera vivido tal número de días». En Epigr. I, 116, 3 la edad de la niña Antula queda indeterminada, pero lo prematuro de su muerte se sugiere con la expresión cito rapta suis, «tempranamente arrebatada a los suyos». El verbo formular vixit se evita y se asocia mediante una imagen poética el invierno con la muerte, contraste entre la rigurosa estación y la floreciente edad de los niños, así en impletura ... sextae ... frigora brumae (Epigr. V, 34, 5); sexta peregit hieme (Epigr. V, 37, 16) «se fue en el sexto invierno»; sextae peremit hiems (Epigr. X, 61, 2) «la mató el sexto invierno»; ultima cui parvae septima venit hiems (Epigr. XI, 91, 2) «para la que vino como último el séptimo invierno».

(b) La filiación sólo aparece explícita en Epigr. VII, 96, 1 y no con el simple genitivo patronímico, sino con la audaz metonimia Bassi dolor, en aposición al nombre del niño: Conditus hic ego sum Bassi dolor, «aquí estoy yo enterrado, el dolor de mi padre Baso». En ella, el sentimiento por su pérdida se toma en lugar del hijo muerto.

(c) La förmula sit tibi terra levis cierra el epitafio V, 34, donde es interesante la utilización del verbo en imperativo con súplica directa a la tierra, tradición helenística ya observada en la Antologia Palatina VII, 461: nec illi, / terra gravis fueris, y la justificación non fuit illa tibi, que pone de relieve la ligereza y pequeñez de la difunta.

La expresión funeraria más habitual hic iacet se modifica cambiando el verbo por otro que tenga unas connotaciones de descanso: Hic festinata requiescit Erotion umbra (Epigr. X, 61, 1), o de protección: Condidit hic natae cineres (Epigr. I, 114, 3); Conditus hic ego, Bassi dolor (Epigr. VII, 96, 1); hoc tegitur cito rapta suis Antulla sepulcro (Epigr. I, 116, 3).

Hay también en Marcial una serie de tópicos propios del epigrama sepulcral que aparecen asimismo en los epitafios infantiles:

- El terreno en que yacen los niños es sagrado: Hoc nemus... sacravit (Epigr. I, 116, 1), y nadie debe turbar su descanso: Si cupit hunc aliquis moneo. ne speret agellum (Epigr. I, 116, 5), «si alguien lo quiere, advierto, no espere este campito».

- Frente a la esterilidad de una muerte infantil, la tierra que cubre al niño es signo de fecundidad. Así, se la califica de hortus (Epigr. I, 114, 1); 
nemus (Epigr. I, 116, 1); caespes (Epigr. V, 34, 9); levis caespes (Epigr. I, 88, 2); sobre la tierra sepulcral debe haber faciles buxos et opacas palmitis umbras (epigr. I, 88, 5), «ágiles bojes y frondosas sombras de sarmiento», en todo caso, producto de nueva vida, terreno de cultivo: culti iugera pulcra soli (Epigr. I, 116, 2), «hermosas yugadas de tierra cultivada», campos que es preciso regar: breve rus udaaue prata (Epigr. I, 114, 2), «un breve campo y húmedas praderas», en ocasiones con la imagen poética del riego con lágrimas: lacrimis roscida prata meis (Epigr. I, 88, 6), «prados regados con mis lágrimas»12.

- Cabe también señalar, como tópico literario en este tipo de epigramas dedicados a niños, la alusión al destino, personificado o no, para justificar muertes tan tempranas: crimine quam fati (Epigr. X, 61, 2), «por culpa del destino»; pessimorum lex amara fatorum (Epigr. V, 37, 15), «la amarga ley de los funestos hados»; debuerant alia fata venire via (Epigr. XI, 91, 10), «debieran por otro camino haber llegado los hados».

- En último lugar, señalamos que, al menos en un caso (Epigr. V, 34), en el epitafio dedicado a la niña Eroción, se nos presenta la figura infantil en el más allá, realizando las actividades que normalmente hubiera hecho en vida:

Hanc tibi, pater, genetrix Flacilla, puellam oscula commendo deliciasque meas, parvola ne nigras horrescat Erotion umbras oraque Tartarei prodigiosa canis.

Impletura fuit sextae modo frigora brumae, vixisset totidem ni minus illa dies.

Inter tam veteres ludat lasciva patronos et nomen blaeso garriat ore meum.

Mollia non rigidus caespes tegat ossa nec illi, terra, gravis fueris: non fuit illa tibi.

*A ti padre Frontón, a ti madre Flacila, encomiendo esta niña, delicia de mis besos:

que no tiemble de miedo la pequeña Eroción ante las negras sombras

y las fauces gigantes del perro del infierno.

Sólo el sexto invierno iba a finar los fríos si ella hubiera vivido tal número de días.

Entre tan viejos dueños retoce juguetona

12 Esta asociación de la muerte con el agua aparece ya en los epitafios griegos y parece tener su origen en la creencia en la sed de los muertos, saciada en la laguna de Lete, que produce el olvido a los que beben de ella. Para este tema y bibliografia sobre el mismo cf. M." L. DEL BARR1O, op. cit., pp. $39-4$ y n. $44,45,46$. 
y que gorjee mi nombre con boca balbuciente, una pradera suave cubra sus tiernos huesos.

Tierra, no seas pesada, ella te fue $\tan$ leve.»

Sin entrar en el tema de la creencia en una vida ulterior, sí es preciso decir que encontramos casos paralelos al señalado para Marcial, en la Antología Palatina, así en $A P$ VII, 482, 5-6 donde se dice que Cleódico, muerto a los tres

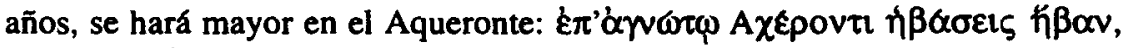

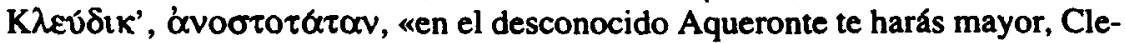
odico, pero sin regreso", y en $A P$ VII, 483, 3-4, donde se considera que el ni-

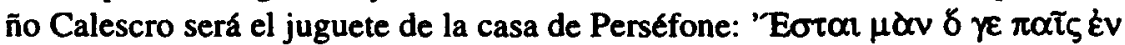

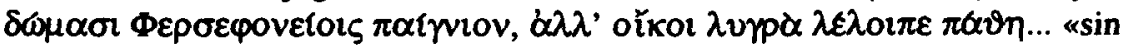
duda, el pequeño será un juguete en la mansión de Perséfone, pero dejó en su casa terrible sufrimiento...»

3. EL EPITAFIO INFANTIL COMO TRADICIÓN CLÁSICA EN AUTORES INGLESES: EL CASO DE LOS POETAS METAFISICOS

Finalmente, encontramos interesantes ejemplos de epitafios dedicados a niños en la literatura inglesa de época isabelina, en concreto dentro de la corriente de los denominados poetas metafísicos ${ }^{13}$. Es conocida la influencia de la literatura clásica en la obra de estos poetas, caracterizada por un estilo difícil, erudito e ingenioso, como es esperable en este grupo de hombres cultos, imbuidos del espíritu de los clásicos grecolatinos. No es extraño, por tanto, encontrar ecos del mundo clásico, también en sus epitafios infantiles, donde, según podemos constatar, se aprecian elementos que continúan la tradición presente en la Antología Palatina y en Marcial.

El primero de los autores que nos ocupan, Ben Jonson (1573-1637), nos presenta el Epitafio para Salathiel Pavy, niño de la Capilla de la Reina Isabel. El título del libro donde dicho poema viene incluido - Epigrams (1616)- es en sí mismo significativo e indica la continuación temática, y en cierta medida también formal, de la literatura epigramática clásica ${ }^{14}$. El mencionado epigrama

13 Cf. T.S. ELLIOT, Los poetas metafisicos y otros ensayos. Buenos Aires, 1944.

14 Las fuentes clásicas en la obra de Jonson es asunto estudiado desde antiguo, cf. W.D. BRIGGS, «Source-Material for Jonson's Epigrams and Forest», Classical Philology 11, 1916, pp. 169-190. Para la 
basa su tratamiento literario en dos elementos fundamentales en la tradición del epitafio infantil, como son la corta edad del difunto y la alusión al destino. Pero una lectura detenida del texto pone en evidencia otros puntos de semejanza en el detalle, así, la propia presentación del tema, haciendo a los lectores una invitación al llanto, que nos recuerda ya los epitafios parlantes griegos y latinos; en este sentido cabe observar el paralelismo entre los versos iniciales del poema (vs. 1-2) weep with me all you that read/this little story y Marcial Epigr. VII, 96, 6 da lacrimas tumulo, qui legis ista, meo. Tras un comienzo de reminiscencias tan clásicas, se presenta el primero de los elementos en que se basa la carga emotiva del texto, la condición infantil del difunto: 'Twas a child (vs. $5)$, expresión sencilla que, como el ya comentado $\tau \partial v \pi \alpha \tau \delta \alpha$ de Calímaco 46,1 , centra el motivo principal del epigrama. También en la precisión con que se señala la edad encontramos un punto de semejanza con el tratamiento literario que de la misma presentaba Marcial, como es el hecho de que para mayor efectividad dramática se consignen los años que el niño no pudo llegar a cumplir: years he numbered scarce thirteen (vs. 9), verso en el que es fundamental el adjetivo scarce «escasos», como en Marcial sexta peregit hieme, nec tamen tota (Epigr. V, 37, 16); sex mihi de prima deerant trieteride menses (Epigr. VII, 96, 3); Impletura fuit sextae modo frigora brumae /vixisset totidem ni minus illa dies (Epigr. V, 34, 5-6) o, con absoluta semejanza en la edad (un adolescente de trece años), el epitafio al liberto Glaucias (Epigr. VI, 28, 11 12): Bis senis modo messibus peractis. vix unum puer adplicabat annum.

El segundo elemento que continúa la tradición grecolatina es la justificación de las muertes prematuras mediante la alusión al destino. Éste aparece personificado en el verso 15 como the Parce, transcripción literal del nombre de las diosas romanas que regían las suertes de los humanos, las Parcas, pronto asimiladas en el mundo romano a las Moiras griegas ${ }^{15}$. Éstas aparecen en perfecto paralelo con el texto de Jonson, en $A P$ VII, 468, 7, precisamente en el

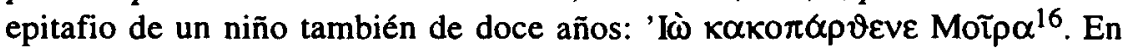

influencia de Marcial en la literatura epigramática moderna, of. A. AMOS, Martial and the Moderns, Cambridge, 1858 y P. NxON, Martial and the modem epigram. New York, 1963; recientemente, J.P. SULLVAN, Martial, the unexpected classic. A literany and historical study, Cambridge, 1991, esp. pp. 288 y ss. ha destacado la influencia de la Antología Palatina y, sobre todo, de la obra de Marcial en los epigramas de Jonson: «onson, glancing perhaps at the tradition of the Greek Anthology, or, more likely, at Martial's broader practice, worked into his Epigrammes not only the expected satirical examples, but also dignified epistles, epitaphs and reflections on life and death, covering the whole range of human experience».

15 Cf. P. Grimal, Diccionario de mitología griega y romana, Barcelona, 1984, s.v. Parcas y J. CONTRERAS et alii, Diccionario de la religión romana, Madrid, 1992, s.v. Parcas.

16 Asimismo, en epitafio epigráfico de Esmima, cf. W. PEEK GV 147, para un niño de once años y para dos hermanos de dos y tres años en un epitafio de Tomis GV 312 . 
este mismo sentido, en los versos 16 y 17 del epigrama de Jonson aparece el termino fate, herencia del fatum latino en la lengua inglesa, en la que, en buena medida, conserva su significado originario ${ }^{17}$. También dentro de este punto cabe incluir el desarrollo temático del niño arrebatado por el más allá a causa de su excesiva bondad y hermosura en los versos 23-24: But being so much too good for earth/Heavens vows to keep him, presente asimismo en uno de los epitafios infantiles de Marcial, en la sentencia: inmodicis brevis est aetas et rara senectus (Epigr. VI, 2, 7), motivo esbozado desde antiguo en la literatura grie-

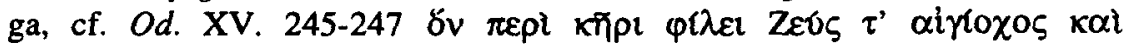

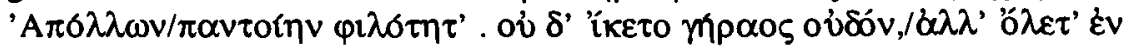

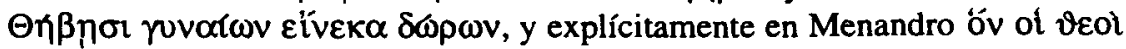

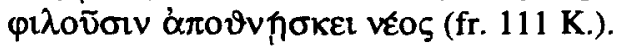

Para finalizar con el texto de Jonson señalemos el motivo del riego, con agua o, metafóricamente, con lágrimas, ya visto en el epigrama funerario latino, y que se asocia, en cualquier caso, a la fecundidad: and have sought (to give new bird) / In baths to steep him (vs. 21-22), en definitiva, nueva alusión al ciclo vida-muerte nueva vida.

Del también poeta isabelino Thomas Carew (1598-1639), discípulo de Jonson, destacamos el epitafio dedicado a una niña (Poems, 1640), en el que asimismo se pueden reconocer elementos tomados de la tradición clásica. Aunque en este epitafio no se consigna el nombre ni los años de la difunta, su edad temprana queda patente en la expresión metafórica 'Twas a bud (vs. 7). En este texto, a diferencia del ya comentado de Ben Jonson, se prescinde de todo tipo de anécdota concreta; se puede decir que todo el poema es un elogio de la belleza Beautie (vs. 2) y del amor Love (vs. 2) que caracterizaban a la niña muerta, a la manera en que aparece en Marcial Epigr. V, 35 la alabanza de las gracias de la niña Eroción.

Asimismo se puede rastrear una semejanza con el motivo de la mors inmatura que veíamos en la Antología Palatina: aquí la niña tampoco ha podido llegar a su plenitud, según se expresa en $A$ budding starre that might have growne / into a Sun, when it had blowne (vs. 9-10) y, aunque no se hace referencia explícita a la ausencia de matrimonio, si se deja entrever que la pequeña ya no podrá inspirar amor a los hombres, así His brand, his bow, let nop man faere / the flammes., the arrowers all lye here (vs. 15-16).

Formalmente, se constata la presencia de elementos clásicos en la expresión formular lye here que aparece en dos ocasiones (vs. 4 y 16). Curiosa es la expresión del verso 4 lyes darkned here por el exacto paralelismo que supone con Marcial Epigr. X, 61, Hic... requiescit Erotion umbra.

17 Cf. OLD s.v. fatum 5: «Fate (regarded as the force which moulds events), destiny». 
El último de los poetas que nos ocupan, Robert Herrick (1591-1674), también discípulo y amigo de Jonson, presenta asimismo en su obra Hesperides varios epitafios al modo clásico, entre los que destacan algunos dedicados a niños donde se ha sugerido en alguna ocasion la influencia de Marcial ${ }^{18}$. Por nuestra parte destacamos dos de ellos dedicados a niñas, en los que la herencia de Marcial y del epitafio grecolatino en general es especialmente visible (los epitafios titulados Upon a child that dyed y Upon a child): ambos se caracterizan por su brevedad, evocando así los epigramas funerarios inscripcionales. La traducción de la fórmula sepulcral latina hic iacet, así como el carácter infantil de las difuntas destacan, en ambos casos, en el primer verso: Here she lies, a Pretty bud (en Upon a child that dyed vs. 1), y Here a Pretty Baby lies (en Upon a child vs. 1). Como Marcial, también Herrick ruega respeto para la tierra sepulcral but not stir / the earth (en Upon a child that dyed vs. 5-6; cf. Marcial Epigr. I, 116, 5). En uno de los casos, como si de una canción de cuna se tratara, ruega silencio: Sung asleep with Lullabies:/ pray be silent and not stir... (en Upon a child vs. 2-3), presentando de nuevo la imagen metafórica del sueño aplicada a la muerte de los niños, como ya vimos en la Antología Palatina VII, 170, 5-619. La tierra, de nuevo considerada como signo de fecundidad y de renovación del ciclo vital, debe regarse: Give her strewins (en Upon a child that dyed vs. 5). Por último, en ambos epitafios la fórmula latina sit tibi terra levis aparece vertida a lengua inglesa en the earth, that lightly covers her (en Upon a child that dyed vs. 6) y th' easy earth that covers her (en Upon a child vs. 4).

En definitiva, hemos pretendido resaltar lo que constituye una herencia grecolatina en la literatura inglesa, que, más allá de simples coincidencias en el tratamiento universal de un tema común, la muerte de los niños, muestra una auténtica tradición literaria.

18 Cf. P.A. Nixon, «Herrick and Martial», Classical Philology 5, 1910, pp. 189-202, esp. 196: «and the arrangement and tone, and sometimes the sentiment, of his epitaphs upon children testify to his appreciation of the tenderness of feeling and delicate perfection of form that occasionally startle us in Martial as would the discovery of a chapel in a divem.

19 Se ha señalado asimismo la influencia de los epigramas de la Antología Palatina (especialmente los de temática amorosa y funeraria) en la literatura epigramática inglesa, y, en concreto, en Herrick. Las numerosas traducciones de los poemas de la Antología, tanto al latín como al inglés, realizadas a partir de la época de Sir Thomas More, facilitarfan el acceso al conocimiento de la obra. La posibilidad de que Herrick conociera algunos de estos epitafios griegos no es, por tanto, descartable; cf. J.A.K. THOMSON, Classical influences on English poetry, Londres, 1951, p. 243: «Herrick would know somethings of these translations, and a large number of the pieces in the Hesperides migth have been suggested by pieces in the Anthology». Tambien Sullivan, op. cit. p.280, ha seffalado junto a la influencia de Marcial, la de la Antología Griega en la obra de Herrick: «Herrick's better known lyrics, on the other hand, reflect the more sentimental and complimentary aspect of Martial's epigrams and the more tender side of the Greek Anthology*. 